\title{
TRAPPING, COMPRESSION AND ACCELERATION OF AN ELECTRON BUNCH IN THE NONLINEAR LASER WAKEFIELD
}

\author{
Arsen G. Khachatryan \\ Yerevan Physics Institute, Alikhanian Brothers Street 2, \\ Yerevan 375036, Armenia
}

\begin{abstract}
A scheme of laser wakefield acceleration, when a relatively rare and long bunch of non-relativistic or weakly-relativistic electrons is initially in front of the laser pulse, is suggested and considered. The motion of test electrons is studied both in the one-dimensional case (1D wakefield) and in the threedimensional laser wakefield excited in a plasma channel. It is shown that the bunch is trapped, effectively compressed both in longitudinal and transverse directions and accelerated to ultra-relativistic energies in the region of first accelerating maximum of the wakefield. The accelerated bunch has sizes much less than the plasma wavelength and relatively small energy spread.
\end{abstract}

PACS numbers: 41.75.Jv, 52.35.Mw, 52.75.Di

\section{INTRODUCTION}

The rapid progress in the technology of high-intensity lasers, based on the chirped-pulse amplification (CPA) [1], opens new opportunities for the use of lasers in many branches of science and industry. Relatively inexpensive tabletop terawatt lasers (so-called $\mathrm{T}^{3}$-lasers) become a qualitatively new tool in physical researches and now are available at many laboratories over the world. Last years CPA technique permitted the production of subpicosecond laser pulses of multiterawatt power with peak intensities exceeding $10^{20} \mathrm{~W} / \mathrm{cm}^{2}$ [2]. With intensities as such we practically have to do with a new interaction range of laser radiation with matter, where the role of the nonlinear effects is often essential. In this intense laser field the matter is usually transformed to plasma and free electrons oscillate with relativistic quivering energy. Presently, the interactions of high-power laser radiation with plasma are actively investigated in connection with different applications: the excitation of strong plasma wake waves for focusing and acceleration of charged bunches [3]; generation of radiation at harmonics of carrier laser frequency [4]; X-ray sources [5]; laser inertial fusion [6] etc.

The laser wakefield, generated in plasma by the short (with the length $\approx \lambda_{p} / 2$, where $\lambda_{p}$ is the plasma wavelength) intense laser pulse provides the acceleration gradient up to tens $\mathrm{GeV} / \mathrm{m}$ (laser wakefield acceleration, LWFA $[7,8])$, that is three orders of magnitude higher than that achieved in conventional accelerators. The main aim of experimental and theoretical works, that are presently in progress, is the construction of compact and relatively inexpensive accelerators of charged particles for applications in 
physics research, medicine and hi-tech industry. However, some challenges remain on this way, one of the main of those is the problem of electron bunch injection.

The wake wavelength in the LWFA is $\lambda_{p} \approx 2 c \tau_{L}$ [3] (here $\tau_{L}$ is the laser pulse duration) and makes up tens or hundreds micrometers for typical plasma densities $n_{p} \sim 10^{16}-10^{19} \mathrm{~cm}^{-3}$. To obtain high quality relativistic electron bunch accelerated by the wake wave, it is necessary to inject short (with the length $L \ll \lambda_{p}$ ), enough dense relativistic electron bunch in the accelerating phase of the wake wave with femtosecond synchronization, that is difficult technical problem (see e.g. Ref. [9]). The injection schemes proposed for the standard LWFA (the LIPA [9], the colliding laser pulses [10] and the LILAC [11] schemes) are aimed at the generation of such a short relativistic bunch.

The diffraction broadening leads to rapid decrease of the intense laser pulse amplitude with the characteristic length $Z_{R}=\pi r_{0}^{2} / \lambda_{L}$ (here $Z_{R}$ is the Rayleigh length, $r_{0}$ is the focal spot size of the pulse, and $\lambda_{L}$ is the laser wavelength) that is typically in order of a millimeter. To prevent diffraction the plasma channel, with minimum density at the axis, proposed to guide the laser pulse in the LWFA [12]. The amplitude of the accelerating component of the wake wave, generated in the plasma channel, decreases as the distance from the laser pulse increases $[13,14]$. Besides, the change of the plasma wavelength $\lambda_{p}$ in the transverse direction $\left[\lambda_{p} \sim n_{p}^{-1 / 2}(r)\right.$, where $n_{p}$ is the density of electrons in the plasma channel], leads to undesirable wave front curving; this effect becomes stronger as the distance from the pulse increases. The effect of the wave front curving in the channel, in the case of a strong laser pulse $\left(a_{0}=e E_{m} / m_{e} c \omega_{L} \geq 1\right.$, where $e$ and $m_{e}$ are the absolute charge and mass of the electron, $E_{m}$ is the maximum amplitude of laser field, $c$ is the speed of light in vacuum, and $\omega_{L}$ is the laser frequency) is amplified due to the nonlinear change of the wake wavelength in transverse direction $[15,16]$. Thus, for regular acceleration of a charged bunch in the wake wave, the most preferable is the region of the first maximum of accelerating field behind the laser pulse.

To avoid the aforesaid difficulties in LWFA, we suggest and study in this work a scheme of trapping, compression and acceleration of a non-relativistic or weakly-relativistic electron bunch in the laser wakefield, when the bunch is initially in front of the laser pulse. The initial bunch density can be much less than that required for the accelerating bunch and the bunch sizes - in order or more than the plasma wavelength, i.e. much more than require other methods of injection [9-11]. Our investigations take into account both the pulse ponderomotive force and the wakefield. It is shown that the bunch is trapped, effectively compressed both in longitudinal and transverse directions and accelerated to ultra-relativistic energies in the region of first accelerating maximum of the wakefield. The accelerated bunch has sizes much less than the plasma wavelength and enough good quality.

\section{THE CASE OF WIDE LASER PULSE}

At first we neglect the transverse variation of the laser pulse amplitude and consider the case of 
one-dimensional laser wakefield excited by a wide bunch in uniform plasma. This allows to study the longitudinal dynamics of the bunch electrons in more details.

\section{A. Basic equations and correlations}

One-dimensional steady wakefield excited by the linearly-polarized laser pulses are described by the following equation (see, e.g. Ref. [3])

$$
\frac{d^{2} \Phi}{d \xi^{2}}+\beta_{g} \gamma_{g}^{2}\left\{1-\beta_{g} \frac{\Phi /\left(1+a^{2} / 2\right)^{1 / 2}}{\left[\Phi^{2} /\left(1+a^{2} / 2\right)-\gamma_{g}^{-2}\right]^{1 / 2}}\right\}=0,
$$

where $\Phi=1+e \varphi / m_{e} c^{2}$ is the dimensionless potential of the plasma wakefield, $a=e E_{0}(\xi) / m_{e} c \omega_{L}, E_{0}$ is the electric field amplitude of the laser pulse, $\xi=k_{p}\left(z-v_{g} t\right), k_{p}=\omega_{p} / v_{g}, \omega_{p}=\left(4 \pi n_{p} e^{2} / m_{e}\right)^{1 / 2}$ is the plasma frequency, $v_{g}$ is the group velocity of the laser pulse which is equal to the phase velocity of the wake wave, $\beta_{g}=v_{g} / c, \gamma_{g}=\left(1-\beta_{g}^{2}\right)^{-1 / 2}$ is the relativistic factor, which, in the case $\gamma_{g}>>1$, is nearly equal to $\omega_{L} / \omega_{p}$. The electric field of exited wakefield, normalized to the non-relativistic wave-breaking field $E_{W B}=m_{e} v_{g} \omega_{p} / e$, can be obtained from equation $E_{z}=-\left(1 / \beta_{g}\right)^{2} d \Phi / d \xi$. The equation of motion of the test electron in the wakefield and in the field of laser pulse is (see e.g. Ref. [17]):

$$
\frac{d p}{d \tau}=-\frac{1}{4 \beta_{g} \gamma} \frac{d a^{2}}{d \xi}-\beta_{g} E_{z}
$$

Here the first term on the right-hand side is the relativistic ponderomotive force averaged over the fast laser oscillations, and the second one corresponds to the plasma wakefield excited by the laser pulse, $\beta=v / c, p=\beta \gamma$ and $\gamma=\left(1+p^{2}+a^{2} / 2\right)^{1 / 2}=\left[\left(1+a^{2} / 2\right) /\left(1-\beta^{2}\right)\right]^{1 / 2}$ are the normalized longitudinal velocity and momentum and the relativistic factor of the test electron (transverse velocity is zero in this Section), $\tau=\omega_{p} t$. Multiplying Eq. (2) by $\beta$, after some simple transformations, one can obtain the following integral of motion (see also Refs. $[14,18]$ )

$$
\gamma-\beta_{g} p-\Phi=\text { const. }
$$

Let us consider an electron that is initially ahead of the laser pulse at some point $\xi_{0}$ where $\Phi=1$ and $a=0$. If the electron has initial momentum $p_{0}<\beta_{g} \gamma_{g}$, it will be overtaken by the laser pulse and can be trapped at some point $\xi_{r}$ inside the pulse or in the wake and accelerated. At the trapping point (or, in other words, at the point of reflection) the velocity of the electron become equal to $v_{g}$. Then from expression (3) we have:

$$
S \equiv\left(1+a_{r}^{2} / 2\right)^{1 / 2} / \gamma_{g}-\left(\Phi_{r}-1\right)=\left(1+p^{2}\right)^{1 / 2}-\beta_{g} p .
$$

In Eq. (4) $a_{r}$ and $\Phi_{r}$ are the amplitude of the laser pulse and the wakefield potential at the reflection point $\xi_{r}$. From Eq. (4) it follows 


$$
p=\gamma_{g}\left[\beta_{g} \gamma_{g} S \pm\left(\gamma_{g}^{2} S^{2}-1\right)^{1 / 2}\right] .
$$

The minus sign in (5) corresponds to the initial momentum $p_{0}$ of an electron which has momentum $\beta_{g} \gamma_{g}$ at the point $\xi_{r}$ and the plus sign corresponds to the momentum of free electron which initially was at the point $\xi_{r}$. Expression (5) describes both trapped and passing particles. In the wake, electrons can be trapped only in the region where $E_{z} \leq 0$. Equation of motion (2) can be rewritten in the form

$$
\frac{d^{2} \xi}{d \tau^{2}}+\frac{\left(1-\beta_{g} \beta\right)}{4 \beta_{g}^{2} \gamma^{2}} \frac{d a^{2}}{d \xi}+\frac{\left(1-\beta^{2}\right)}{\gamma} E_{z}=0
$$

where $\xi$ is the coordinate of a test electron in the frame commoving with the laser pulse. The dimensionless velocity of the electron one can obtain from expression $\beta=\beta_{g}(1+d \xi / d \tau)$.

\section{B. Numerical results}

Eqs. (1) and (6) were solved numerically for the Gaussian laser pulse,

$$
a=a_{0} \exp \left[-\left(\xi-\xi_{c}\right)^{2} / \sigma_{z}^{2}\right]
$$

In Fig. 1 a laser pulse with $a_{0}=2$ and nonlinear wakefield excited by it are presented (here and below in numerical calculations $\sigma_{z}=2, \xi_{c}=3$ and $\gamma_{g}=10$ ). The amplitude of the wake wave is essentially less than one-dimensional relativistic wave-breaking field $E_{r e l}=\left[2\left(1-\gamma_{g}\right)\right]^{1 / 2} / \beta_{g} \approx 4.26[3,19]$. Figure 2 shows the dependence of initial electron momentum $p_{0}$ on the trapping point near the first accelerating maximum in the wake wave. The minimum value of the initial momentum $p_{\min }$ corresponds to the trapping point where the potential is at the minimum and $E_{z}=0$. Curves 1 and 2 in Fig. 2 reach their minimums at different points, that is the consequence of the nonlinear increase of wake wavelength with the amplitude (this dependence can be found in Ref. [20]). The curves were obtained numerically and coincide with the expression (5) for the trapped particles. Fig. 3 shows the dependence of the value of $p_{\min }$ and wake wave amplitude $E_{z, \max }$ on $a_{0}$. One can see that a laser pulse with $a_{0} \sim 1$ (that corresponds to the peak intensity of the pulse $I_{\max } \sim 10^{18} \mathrm{~W} / \mathrm{cm}^{2}$ for $\lambda_{L}=1 \mu \mathrm{m}$, and $I_{\max } \sim 10^{16} \mathrm{~W} / \mathrm{cm}^{2}$ when $\left.\lambda_{L}=10 \mu \mathrm{m}\right)$ provides trapping of initially non-relativistic or weakly-relativistic electrons in the wake wave. For example, $p_{\min } \approx 0.4$ for the wakefield presented in Fig. 1. Electrons with $p_{0}<p_{\min }$ can not be trapped in the wake wave and may be detected behind the wave. This circumstance can help to determine the wake wave amplitude in experiments. Our numerical calculations have witnessed that electrons with $p_{0}<\beta_{g} \gamma_{g}$ can not be trapped in the region occupied by the laser pulse because of the decelerating wakefield; only electrons with $p_{0} \approx \beta_{g} \gamma_{g}$ are trapped in the head of the pulse (where $\left.E_{z} \approx 0\right)$ due to the ponderomotive force. This confirms with the results of Ref. [18].

Figure 4 shows the behavior of electrons of mono-energetic non-relativistic $\left(p_{0}=0.5, \gamma_{0}=(1+\right.$ $\left.p_{0}^{2}\right)^{1 / 2} \approx 1.12$ ) bunch in the wakefield presented in Fig. 1 . Initial dimensionless bunch length is $L_{0}=5$, 
that roughly corresponds to the linear plasma wavelength $\lambda_{p}$. When $\tau=50$, the trapped bunch length is $L \approx 0.027$ and $L \approx 0.04$ when $\tau=100$, that is two orders of magnitude less than the initial bunch length. The absolute energy spread $\Delta \gamma$ in the accelerating bunch increases insignificantly with time, but the relative energy spread $\varepsilon=\Delta \gamma / \gamma$ falls due to growing $\gamma$; for example $\varepsilon \approx 0.26$ at $\tau=50$, and $\varepsilon \approx 0.14$ when $\tau=100$. The acceleration gradient in the considering case is approximately equal to $2 \mathrm{MeV} / \lambda_{p}$. For example, when $\lambda_{p}=100 \mu m\left(n_{p} \approx 10^{17} \mathrm{~cm}^{-3}\right)$, the acceleration gradient is $20 \mathrm{GeV} / \mathrm{m}$.

Figure 5 shows the motion of electrons with different initial momentums and the same initial positions $\left(0.6 \leq p_{0} \leq 1.2,1.17 \leq \gamma \leq 1.56, \xi_{0}=0\right)$ in the wakefield presented in Fig. 1. The trapped bunch length is nearly 27 times less than the plasma wavelength $\lambda_{p}$. The relative energy spread at $\tau=100$ is about 0.1 , that is much less than that of initial electrons.

The dephasing length, for electrons with $p_{\min } \leq p_{0} \leq 1.2$, varies in the range $630 \leq L_{d} \leq 700$ (the grater values correspond to the smaller initial momentums) that is comparable with the linear dephasing length $\lambda_{p} \gamma_{g}^{2}$ [3], which, in our notations, corresponds to $L_{d}=2 \pi \gamma_{g}^{2}=200 \pi$. The maximum relativistic factor of accelerated particles is in the range $350 \leq \gamma_{\max } \leq 410$ (here again the greater values correspond to smaller $p_{0}$ ) that essentially exceeds the linear value $2 \gamma_{g}^{2}=200$ [3], but is an order of magnitude less than the maximum nonlinear value $4 \gamma_{g}^{3}=4000[20,21]$.

\section{Energy spread in the accelerating bunch}

Energy spread in the trapped bunch depends on energy spread and length of the initial bunch. The tail electrons of initial bunch are trapped earlier and therefore, have greater energy during acceleration (see Fig. 4). Slower particles also are trapped earlier (see Fig. 5). Let us suppose that initially the bunch is at the head of laser pulse, so that $\xi=0$ corresponds to the bunch tail, and $\tau_{t r}\left(p_{0}\right)$ is the time necessary to trap an electron which is initially at $\xi=0$; the initial electron momentum is in the range $p_{1} \leq p_{0} \leq p_{2}$. Then, for energy spread in the trapped bunch we can write $\Delta \gamma \sim \Delta \tau_{t r} E_{z, \max }=$ $\left[\tau_{t r}\left(p_{2}\right)-\tau_{t r}\left(p_{1}\right)+L_{0} /\left(1-v_{2} / v_{g}\right)\right] E_{z, \max }$, where $\Delta \tau_{t r}$ is the time interval which is necessary to trap the initial bunch. For the relative energy spread one has: $\varepsilon \sim \Delta \tau_{t r} /\left(\tau-\Delta \tau_{t r}\right)$. These estimates agree well with the numerical results. One can see that the presence of fast electrons (with $v_{0} \sim v_{g} \approx c$ ) in the initial bunch leads to an undesirable increase in the energy spread.

The trapped bunch density can be found from expression $n_{b}(\tau) \approx n_{b 0} L_{0} / L(\tau)$, where $n_{b 0}$ is the initial bunch density.

\section{Wakefield generated by the accelerating bunch}

The trapped bunch also generates wakefield which can destroy the laser wakefield and decrease the accelerating field. Because the accelerating bunch is short $\left(L(\tau) \ll \lambda_{p}\right)$ we can consider it as a plane bunch and find the normalized amplitude of the wakefield excited by the bunch from expression $E_{b, \max }=$ 
$k_{p}\left(v_{b} / c\right)\left(N_{b} / n_{p}\right)$ [22], where $v_{b}$ and $N_{b}$ are velocity and the surface density of the bunch correspondingly. This expression is valid both in linear and non-linear regimes. In our case $N_{b}=\delta n_{b 0} L_{0} / k_{p}$, where $\delta \leq 1$ is the ratio of number of trapped electrons to the total number of particles in the initial bunch, and we have:

$$
E_{b, \max }=\delta\left(v_{b} / c\right)\left(n_{b 0} L_{0} / n_{p}\right)
$$

The normalized amplitude of moderately nonlinear laser wake wave, considering in this paper, is about unit. So, we can neglect the wakefield generated by the bunch if $E_{b, \max } \ll 1$, or when

$$
n_{b 0} \ll n_{p}\left(c / v_{b}\right)\left(1 / \delta L_{0}\right) .
$$

For $n_{p} \sim 10^{16}-10^{18} \mathrm{~cm}^{-3}$ (that is typical for the LWFA experiments [3]), $v_{b} \approx c, \delta \approx 1$ and the initial bunch length in order of $\lambda_{p}\left(L_{0} \sim 5-10\right)$ this condition reads $n_{b 0}<10^{14}-10^{16} \mathrm{~cm}^{-3}$. The density of accelerating bunch may be in order of plasma density.

Thus, the one-dimensional analysis has showed the possibility of trapping, essential compression and high-gradient acceleration of a low energy electron bunch in moderately nonlinear laser wakefield.

\section{TRAPPING, COMPRESSION AND ACCELERATION IN THE LASER WAKEFIELD EXCITED IN PLASMA CHANNEL}

In this section we consider our scheme of LWFA for the case of laser wakefield excited in a plasma channel and study the peculiarities of radial motion of test electrons during trapping and acceleration.

\section{A. Nonlinear laser wakefield excited in plasma channel}

As was mentioned in Introduction, the plasma channel is necessary to guide a laser pulse. This allows to essentially increase the laser-plasma interaction distance [12], that, in its turn, provides ultrarelativistic acceleration in the wakefield [3]. Nonlinear axially-symmetrical laser wakefields excited in a plasma channel are described by the following system of equations [15]:

$$
\begin{gathered}
\beta \frac{\partial p_{z}}{\partial \xi}-\frac{\partial \gamma_{e}}{\partial \xi}-\beta^{2} E_{z}=0, \\
\beta \frac{\partial p_{r}}{\partial \xi}-\frac{\partial \gamma_{e}}{\partial r}-\beta^{2} E_{r}=0 \\
-\frac{\partial H_{\theta}}{\partial \xi}+\beta \frac{\partial E_{r}}{\partial \xi}+\beta_{r} N_{e}=0 \\
\nabla_{\perp} H_{\theta}+\beta \frac{\partial E_{z}}{\partial \xi}+\beta_{z} N_{e}=0
\end{gathered}
$$




$$
\begin{gathered}
\beta \frac{\partial H_{\theta}}{\partial \xi}-\frac{\partial E_{r}}{\partial \xi}+\frac{\partial E_{z}}{\partial r}=0, \\
N_{e}=N_{p}(r)-\nabla_{\perp} E_{r}-\frac{\partial E_{z}}{\partial \xi},
\end{gathered}
$$

where $E_{z, r}$ and $H_{\theta}$ are longitudinal and radial components of the electric field and azimuthal component of the magnetic field normalized to the on-axis wave-breaking field $E_{W B}(r=0)=m_{e} \omega_{p}(r=0) v_{g} / e$, $p_{z, r}$ are the normalized components of plasma electron momentum, $\gamma_{e}=\left(1+p_{z}^{2}+p_{r}^{2}+a^{2} / 2\right)^{1 / 2}$ is the relativistic factor, $\beta_{z, r}=p_{z, r} / \gamma_{e}, N_{e}=n_{e}(\xi, r) / n_{p}(0)$ is the normalized density of plasma electrons, $n_{p}(r)$ is unperturbed plasma density in the channel, $N_{p}=n_{p}(r) / n_{p}(0), \nabla_{\perp}=\partial / \partial r+1 / r$. The force acting on the relativistic electrons in the wakefield is $\mathbf{F}\left(-\mathbf{e E}_{\mathbf{z}},-\mathbf{e}\left(\mathbf{E}_{\mathbf{r}}-\boldsymbol{\beta} \mathbf{H}_{\boldsymbol{\theta}}\right), \mathbf{0}\right)$. According to (8.5)

$$
\frac{\partial E_{z}}{\partial r}=\frac{\partial\left(E_{r}-\beta H_{\theta}\right)}{\partial \xi} \equiv-\frac{\partial f_{r}}{\partial \xi} .
$$

So, the field of forces $\mathbf{F}$ is potential because $\nabla \times \mathbf{F}=\mathbf{0}$, and one can write $\mathbf{F}=\nabla \boldsymbol{\Phi}(\boldsymbol{\xi}, \mathbf{r})$, here $\Phi=1-\int_{\xi}^{0} E_{z} d \xi$.

In this section we consider an axially-symmetric laser pulse which has Gaussian profile both in longitudinal and radial directions:

$$
a(\xi, r)=a_{0} \exp \left[-\left(\xi-\xi_{c}\right)^{2} / \sigma_{z}^{2}\right] \exp \left(-r^{2} / \sigma_{r}^{2}\right)
$$

The laser pulse is guided in preformed plasma channel which has the following unperturbed electron density:

$$
N_{p}=\left(1+\Delta \frac{r^{2}}{r_{c h}^{2}}\right) \exp \left(-b \frac{r^{4}}{r_{c h}^{4}}\right)
$$

where $r_{c h}, \Delta$ and $b \ll 1$ are constant values. Such a density profile is typical for plasma channels created in experiments [23]. Suppose that the pulse is guiding without change in its radius $\sigma_{r}$. In this case $\sigma_{r}=r_{c h}$ and $n_{p}\left(r_{c h}\right)-n_{p}(0)=1 / \pi r_{e} r_{c h}^{2}$, where $r_{e}=e^{2} / m_{e} c^{2} \approx 2.8 \times 10^{-13} \mathrm{~cm}$ is the classical electron radius and all values are dimensional [12]. Then, in expression (10), $\Delta=\left(2 / \sigma_{r} \beta_{g}\right)^{2}$.

Equations (8.1)-(8.6) were solved numerically for the following parameters of the problem: $a_{0}=2$, $\sigma_{z}=2, \sigma_{r}=5$ and $\gamma_{g}=10$. In this case $\Delta \approx 0.16$, the value of $b$ was chosen to be 0.01 . In Fig. 7 we present the radial profile of unperturbed plasma density and the radial behavior of laser pulse intensity, namely $\exp \left(-2 r^{2} / \sigma_{r}^{2}\right)$. Fig. 8 shows the longitudinal electric field and the focusing field $f_{r}=\beta H_{\theta}-E_{r}$ of the wakefield excited. One can see that the wake wavelength decreases as $r$ increases. This is caused by the radial increase of unperturbed plasma density in the channel $[3,13]$ and by the nonlinear increase of wavelength with the wake wave amplitude which is at maximum on the axis [15,16,24]. Fig. 7 shows also the nonlinear steepening of the accelerating field like that takes place in one-dimensional wakefield (see Fig. 1). Due to the dependence of the wavelength on $r$, the field in the radial direction grows more 
chaotic as the distance from the laser pulse increases. In fact, the oscillations of the plasma for different $r$ are started behind the pulse with nearly equal phases but different wavelengths. As $|\xi|$ increases, the change of phase in the transverse direction becomes more and more marked. This leads to a curving of the phase front and to oscillations in the transverse direction $[15,16,24]$. Such behavior of the wakefield excited in a plasma channel leads to the transverse multistream motion of plasma electrons in the wake and to the transverse wave-breaking [25]. The radial dependence of longitudinal electric field and the focusing force is shown in Fig. 8 for point $\xi=-10.9$ at which the on-axis accelerating field reaches its maximum. We see that the wakefield changes its sign and is steepened. For the ultra-relativistic acceleration of electrons one needs to use a region in the wakefield where the conditions $E_{z}<0$ and $f_{r}<0$ are satisfied simultaneously. The radial steepening leads to the radial restriction or the region suitable for acceleration. Near the first accelerating maximum of the wakefield shown in Fig. 7, the suitable region is $r<2.8$. As the distance from the laser pulse increases, the suitable region becomes narrower, so that at some distance the wakefield is highly irregular. Thus, the most preferable for electron acceleration is the region of the first accelerating maximum in the wake.

\section{B. Equation of motion of bunch electrons}

Three-dimensional vector equation of motion of bunch electrons is

$$
\frac{d \mathbf{p}}{d \tau}=-\beta_{g}(\mathbf{E}+\boldsymbol{\beta} \times \mathbf{H})-\frac{\mathbf{1}}{\mathbf{4} \boldsymbol{\beta}_{\mathbf{g}} \gamma} \nabla \mathbf{a}^{2} .
$$

Here all values are dimensionless, $\boldsymbol{\beta}=\mathbf{v} / \mathbf{c}=\mathbf{p} / \boldsymbol{\gamma}$ is the normalized velocity, $\gamma=\left(1+\mathbf{p}^{\mathbf{2}}+\mathbf{a}^{\mathbf{2}} / \mathbf{2}\right)^{\mathbf{1} / \mathbf{2}}=$ $\left[\left(\mathbf{1}+\mathbf{a}^{\mathbf{2}} / \mathbf{2}\right) /\left(\mathbf{1}-\boldsymbol{\beta}^{\mathbf{2}}\right)\right]^{\mathbf{1} / \mathbf{2}}$ is the relativistic factor. For the momentum components, from Eq. (11) one has:

$$
\begin{gathered}
\frac{d p_{r}}{d \tau}=-\beta_{g}\left(E_{r}-\beta_{z} H_{\theta}\right)-\frac{1}{4 \beta_{g} \gamma} \frac{\partial a^{2}}{\partial r}, \\
\frac{d p_{\theta}}{d \tau}=0 \\
\frac{d p_{z}}{d \tau}=-\beta_{g}\left(E_{z}+\beta_{r} H_{\theta}\right)-\frac{1}{4 \beta_{g} \gamma} \frac{\partial a^{2}}{\partial \xi} .
\end{gathered}
$$

It follows from Eq. (12.2) that the azimuthal momentum is conserved, $p_{\theta}=$ const, $\beta_{\theta}(\tau)=p_{\theta}(0) / \gamma(\tau)$. The azimuthal momentum has not essential influence on the axial and radial dynamics, and we suppose $p_{\theta}(0)=0$ in this paper. For the energy of electrons Eq. (11) gives the following equation:

$$
\frac{d \gamma}{d \tau}=-\beta_{g}(\boldsymbol{\beta E})-\frac{1}{4 \gamma} \frac{\partial \mathbf{a}^{2}}{\partial \boldsymbol{\xi}}
$$


From Eqs. (12.4), (13) and (9) we obtain the integral of motion

$$
\gamma-\beta_{g} p_{z}-\Phi(\xi, r)=\text { const }
$$

which formally coincides with the one-dimensional integral of motion (3) [14,26,27]. Electrons can be trapped in the region where wakefield is both accelerating and focusing. For the scattered particles, from Eq. (14) one has $p_{r}^{2}=\left(S+\beta_{g} p_{z}\right)^{2}-p_{z}^{2}-1$, here $S=\left[1+\mathbf{p}^{\mathbf{2}}(\mathbf{0})\right]^{\mathbf{1} / \mathbf{2}}-\boldsymbol{\beta}_{\mathbf{g}} \mathbf{p}_{\mathbf{z}}(\mathbf{0})$. If an electron is initially non-relativistic $(|\mathbf{p}(\mathbf{0})| \ll \mathbf{1}, S \approx 1)$, then $p_{r} \approx\left(2 p_{z}\right)^{1 / 2}$ and $\tan \theta=p_{r} / p_{z} \approx[2 /(\gamma-1)]^{1 / 2}$, where $\theta$ is the angle between $z$-axis and final momentum of the scattered electron [28].

Taking into account Eq. (13), we rewrite Eqs. (12.1) and (12.3) in the form

$$
\begin{gathered}
\frac{d^{2} \xi}{d \tau^{2}}+\frac{1}{\gamma}\left[\left(1-\beta_{z}^{2}\right) E_{z}-\beta_{z} \beta_{r} E_{r}+\beta_{r} H_{\theta}\right]+\frac{\left(1-\beta_{g} \beta_{z}\right)}{4 \beta_{g}^{2} \gamma^{2}} \frac{\partial a^{2}}{\partial \xi}=0, \\
\frac{d^{2} r}{d \tau^{2}}+\frac{1}{\gamma}\left[\left(1-\beta_{r}^{2}\right) E_{r}-\beta_{z} \beta_{r} E_{z}-\beta_{z} H_{\theta}\right]-\frac{1}{4 \beta_{g} \gamma^{2}}\left(\beta_{r} \frac{\partial a^{2}}{\partial \xi}-\frac{1}{\beta_{g}} \frac{\partial a^{2}}{\partial r}\right)=0 .
\end{gathered}
$$

The normalized components of velocity obey the formulae $\beta_{z}=\beta_{g}(1+d \xi / d \tau)$ and $\beta_{r}=\beta_{g} d r / d \tau$. For particles trapped in the wakefield, we suppose that during acceleration $\beta_{z} \approx 1, \beta_{r}^{2} \ll 1$ and $r<1$ (the numerical results presented below show that this is the case). Then, from Eq. (15.1) one has

$$
d^{2} \xi / d \tau^{2} \approx E_{z} / \gamma^{3}
$$

It follows from this equation that $d \gamma / d \tau \approx-E_{z}$ and $\gamma \approx-\int E_{z} d \tau$. Thus, the longitudinal dynamics of accelerating particles is approximately the same as in the one-dimensional case. The radial motion of electrons, according to Eq. (15.2), obeys the equation

$$
\frac{d^{2} r}{d \tau^{2}}+\frac{\left|E_{z}\right|}{\gamma} \frac{d r}{d \tau}+\Omega^{2} r \approx 0
$$

where $\Omega=\left(\left|\partial f_{r} / \partial r\right| / \gamma\right)^{1 / 2}$ is the betatron frequency. Supposing that the value of $E_{z}$ is approximately conserved during acceleration, we can write $\gamma \approx\left|E_{z}\right|\left(\tau-\tau_{t r}\right)$. In this case solution of Eq. (17) is

$$
r=r\left(\tau_{t r}\right) J_{0}\left[2\left(\left|\partial f_{r} / \partial r\right|\left(\tau-\tau_{t r}\right) /\left|E_{z}\right|\right)^{1 / 2}\right],
$$

where $J_{0}$ is the Bessel function of zero order.

\section{Results of test-particle simulations and discussion}

Motion of test electrons in the 2D wakefield presented in Fig. 7 was investigated by numerical solution of Eqs. (15.1) and (15.2) for different initial positions and momentums. Figure 9 shows the behavior of electrons with zero initial transverse momentums and with different initial radial positions. One can 
see that particles are trapped near the first accelerating maximum in the wake. During the trapping, electrons concentrate near the axis due to the focusing force $\beta_{z} H_{\theta}-E_{r}$. Sins the longitudinal size of the trapped bunch is much less than the plasma wavelength and its transverse size is essentially less than that of the laser pulse, the electrons experience approximately the same accelerating field; the longitudinal dynamics is well described by the one-dimensional theory. The focusing force acting on the bunch electrons depends on $r$ linearly (see Fig. 8). The small bunch sizes (as compared with the wakefield characteristic sizes) and the fact that electrons are trapped near the accelerating maximum provide high accelerating gradient and relatively small energy spread. For example, the relative energy spread of electrons presented in Fig. 9 is $5 \%$ at $\tau=300$. The numerical results show that dynamics of the accelerating bunch is well described by approximate equations (16)-(18). The betatron oscillations of the accelerating electrons are clearly seen in Fig. 9(b). The wavelength of this oscillations decreases with the increase of particle's energy that conform to the formula for betatron frequency. Radial velocity of accelerating electrons is much less than the longitudinal one, $\left|\beta_{r}(\tau)\right|<0.1$. One can see also that even electrons which are initially at the periphery $\left[r(\tau=0) \equiv r_{0} \sim \sigma_{r}\right]$ can be trapped in the wakefield and accelerated. The characteristic dependence of the minimum trapping threshold $p_{z, \text { min }}$ on the initial radial position of an electron is presented in Fig. 10. Figure 11 shows the minimum and maximum initial radial momentums of trapped electrons in dependence on initial radial position. The figure witnesses that electrons which initially move at relatively high angle to the axis (up to tens degrees) also can be trapped and accelerated. This again is caused by the focusing force and the fact that electrons are initially non-relativistic $\left(\gamma_{0} \sim 1\right)$.

In Fig. 12 we show behavior of electrons of a bunch with the following initial parameters: $0 \leq \xi_{0} \leq 5$, $r_{0} \leq 4,0.6 \leq p_{z 0} \leq 0.8,-0.02 \leq p_{r 0} \leq 0.02$. The passing particles (not showed) are well separated from accelerating one both spatially and energetically. The length of accelerating bunch in this case also is much less than the plasma wavelength $[L(\tau=100) \approx 0.27, L(\tau=300) \approx 0.19]$. The radius of the bunch $R$ decreases relatively slowly during acceleration and is essentially less than the characteristic transverse size of the wakefield $\sigma_{r}, R(\tau) \sim 1$; the bunch radius can be reduced by the choice of smaller laser spot size. The absolute energy spread does not change practically, $\Delta \gamma \approx 24$, but the relative energy spread falls and is equal to about $10 \%$ at $\tau=300$. The estimations of absolute and relative energy spreads presented in Sec. II are valid also in $3 \mathrm{D}$ case.

Total number of electrons trapped and their density can be estimated from expressions

$$
\begin{gathered}
N_{t o t} \sim \delta \pi n_{b 0} \sigma_{r}^{2} L_{0} / k_{p}^{3}, \\
n_{b} \sim \delta n_{b 0}\left(\sigma_{r} / R\right)^{2}\left(L_{0} / L\right) .
\end{gathered}
$$


The on-axis amplitude of the linear wake wave excited by the bunch is reduced by the factor $T(R)=$ $1-R K_{1}(R)<1$ [29] (where $K_{1}$ is the modified Bessel function) as compared to the one-dimensional case (see Sec. II). Therefore, in our case, for the amplitude of wakefield generated by the bunch, we have: $E_{b, \max } \approx T L n_{b} / n_{p}$. This wakefield can be neglected when $E_{b, \max } \ll E_{z, \max }$, or taking into account (20) - if $\delta T L_{0}\left(\sigma_{r} / R\right)^{2}\left(n_{b 0} / n_{p}\right) \ll 1$; when $R \ll 1, T \approx R^{2} / 2$, and this condition reads $\delta L_{0} \sigma_{r}^{2} n_{b 0} / 2 n_{p} \ll 1$. Total number of bunch electrons, according to (19), is restricted by the following condition: $N_{t o t} \ll$ $\pi n_{p} k_{p}^{-3}\left(R^{2} / T\right) \approx 1.4 \times 10^{7}\left(R^{2} / T\right) \lambda_{p}[\mu m]$.

For the normalized emittance $\varepsilon_{n}=\sigma_{0}^{2} / \beta$ (here $\sigma_{0}$ is the matched transverse size of the bunch, $\beta$ is the betatron length) of the accelerating bunch, in our notations, one can write $\varepsilon_{n} \sim R^{2} \Omega \lambda_{p} / 4 \pi^{2}$. In the case $\lambda_{p}=100 \mu m\left(n_{p} \approx 10^{17} \mathrm{~cm}^{-3}\right)$, for the bunch presented in Fig. $12, \varepsilon_{n} \sim 8 \mathrm{~nm} / \gamma^{1 / 2}$; for example, $\varepsilon_{n} \sim 0.5 \mathrm{~nm}$ when $\gamma=300$, that is comparable with the emittance expected in the TeV-range laser wakefield accelerator [30,31] (see also Refs. [14,31-34] for the dynamics of accelerating bunch).

\section{SUMMARY}

The results of the present work show the possibility of trapping, essential compression both in longitudinal and transverse directions and ultra-relativistic acceleration of an initially non-relativistic or weakly-relativistic electron bunch in moderately nonlinear $\left(a_{0} \sim 1, E_{z, \max } \sim 1\right)$ laser wakefield. The initial bunch can be generated, for example, by a photocathode. So far as electron bunch is initially non-relativistic $\left(\gamma_{0} \sim 1\right)$, trapping and compression take place during time interval comparable with the plasma wave period, that is much less than the time scale of longitudinal dynamics of relativistic particles in the wake [33]. Due to the fact that trapped bunch sizes are essentially less than characteristic spatial scales of the wake wave, the energy spread in the accelerated bunch can be relatively low, namely few percent. In our scheme the problems connected with the wake wavefront curvature also are removed. The electron bunch trapped and accelerated can be accelerated further in the multi-stage LWFA [31].

Thus, the scheme of LWFA proposed, has the following advantages: (a) instead of injection of an enough dense relativistic electron bunch with small sizes (in order of a micrometer), our scheme utilizes a non-relativistic, rare and long electron bunch, that is much easier to get technically, (b) femtosecond electron bunch synchronization in the laser wakefield is not required, (c) effective electron bunch compression, and (d) spatial and energetic separation of the initial electron bunch, that can decrease the trapped bunch emittance.

\section{ACKNOWLEDGMENT}

The author is grateful to B. Hafizi, R. Hubbard and P. Sprangle (Naval Research Laboratory, Washington, DC) for helpful discussions.

\section{REFERENCES}


[1] D. Strickland and G. Mourou, Opt. Commun. 56, 219 (1985).

[2] G. A. Mourou, C. P. Barty, and M. D. Perry, Phys. Today 51, 22 (1998).

[3] E. Esarey, P. Sprangle, J. Krall, and A. Ting, IEEE Trans. Plasma Sci. 24, 252 (1996).

[4] P. Sprangle, E. Esarey, and A. Ting, Phys. Rev. A 41, 4463 (1990).

[5] N. E. Burnett and P. B. Corkum, J. Opt. Soc. Am. B 6, 1195 (1989).

[6] M. Tabak, J. Hammer, M. E. Glinsky, W. L. Kruer, S. C. Wilks, J. Woodworth, E. M. Campbell, M. D. Perry, and R. J. Mason, Phys. Plasmas 1, 1626 (1994).

[7] T. Tajima and J. M. Dawson, Phys. Rev. Lett. 43, 267 (1979).

[8] L. M. Gorbunov and V. I. Kirsanov, Zh. Eksp. Teor. Fiz. 93, 509 (1987) [Sov. Phys. JETP 66, $290(1987)]$.

[9] C. I. Moore, A. Ting, S. J. McNaught, J. Qiu, H. R. Burris, and P. Sprangle, Phys. Rev. Lett. 82, 1688 (1999).

[10] D. Umstadter, J. K. Kim, and E. Dodd, Phys. Rev. Lett. 76, 2073 (1996).

[11] E. Esarey, R. F. Hubbard, W. P. Leemans, A. Ting, and P. Sprangle, Phys. Rev. Lett. 79, 2682 (1997); E. Esarey, C. B. Schroder, W. P. Leemans, and B. Hafizi, Phys. Plasmas 6, 2262 (1999).

[12] E. Esarey, P. Sprangle, J. Krall, and A. Ting, IEEE J. Quantum Electron. 33, 1879 (1997).

[13] N. E. Andreev, L. M. Gorbunov, V. I. Kirsanov, K. Nakajima, and A. Ogata, Phys. Plasmas 4, 1145 (1997).

[14] A. J. W. Reitsma, V. V. Goloviznin, L. P. J. Kamp, and T. J. Schep, Phys. Rev. E 63, 046502 (2001).

[15] A. G. Khachatryan, Phys. Rev. E 60, 6210 (1999).

[16] A. G. Khachatryan, Phys. Plasmas 7, 5252 (2000).

[17] G. Shvets, N. J. Fisch, and A. Pukhov, IEEE Trans. Plasma Sci. 28, 1194 (2000).

[18] C. Du and Z. Xu, Phys. Plasmas 7, 1582 (2000).

[19] A. I. Akhiezer and R .V. Polovin, Zh. Eksp. Teor. Fiz. 30, 915 (1956) [Sov. Phys. JETP 3, 696 (1956)].

[20] A. G. Khachatryan, Phys. Plasmas 4, 4136 (1997).

[21] E. Esarey and M. Pilloff, Phys. Plasmas 2, 1432 (1995).

[22] R. D. Ruth, A. W. Chao, P. L. Morton, and P. B. Wilson, Part. Accel. 17, 171 (1985).

[23] A. J. Mackinnon, M. Borghesi, A. Iwase, and O. Willi, Phys. Rev. Lett. 80, 5349 (1998); E. De Wispelaere, V. Malka, S. Hüller, F. Amiranoff, S. Baton, R. Bonadio, M. Casanova, F. Dorchies, R. Haroutunian, and A. Modena, Phys. Rev. E 59, 7110 (1999); G. S. Sarkisov, V. Yu. Bychenkov, V. N. Novikov, V. T. Tikhonchuk, A. Maksimchuk, S.-Y. Chen, R. Wagner, G. Mourou, and D. Umstadter, ibid. 59, 7042 (1999); T. R. Clark and H. M. Michberg, Phys. Plasmas 7, 2192 (2000); J. Faure, V. Malka, J.-R. Marquès, F. Amiranoff, C. Courtois, Z. Najmudin, K. Krushelnick, M. Salvati, A. E. 
Dangor, A. Solodov, P. Mora, J.-C. Adam, and A. Héron, ibid. 7, 3009 (2000); E. W. Gaul, S. P. Le Blanc, A. R. Rundquist, R. Zgadzaj, H. Langhoff, and M. C. Downer, Applied Phys. Lett. 77, 4112 (2000).

[24] A. G. Khachatryan, Fizika Plazmy 27, 921 (2001) [Plasma Phys. Rep. 27, 860 (2001)].

[25] S. V. Bulanov, F. Pegoraro, A. M. Pukhov, and A. S. Sakharov, Phys. Rev. Lett. 78, 4205

(1997); S. V. Bulanov, F. Pegoraro, and J. Sakai, Nucl. Instrum. Methods Phys. Res. A 410, 477 (1998).

[26] P. Mora and T. M. Antonsen, Phys. Rev. E 53, R2068 (1996).

[27] S.-Y. Chen, M. Krishnan, A. Maksimchuk, and D. Umstadter, Phys. Plasmas 6, 4739 (1999).

[28] P. B. Corcum, N. H. Burnett, and F. Brunel, in Atoms in Intense Fields, edited by M. Gavrila (Academic Press, New York, 1992).

[29] R. Keinigs and M. Jones, Phys. Fluids 30, 252 (1987); A. G. Khachatryan, A. Ts. Amatuni, E. V. Sekhposyan, and S. S. Elbakyan, Fizika Plazmy 22, 638 (1996) [Plasma Phys. Rep. 22, 576 (1996)]. [30] M. Xie, T. Tajima, K. Yokoya, and S. Chattopadhyay, in Advanced Accelerator Concepts: Seventh Workshop, Lake Tahoe, 1996, edited by S. Chattopadhyay (AIP, New York, 1997), p. 233.

[31] S. Cheshkov, T. Tajima, and W. Horton, Phys. Rev. ST-AB 3, 071301 (2000).

[32] R. Assman and K. Yokoya, Nucl. Instrum. Methods Phys. Res. A 410, 544 (1998).

[33] N. E. Andreev, S. V. Kuznetsov, and I. V. Pogorelsky, Phys. Rev. ST-AB 3, 021301 (2000).

[34] C. Chiu, S. Cheshkov, and T. Tajima, Phys. Rev. ST-AB 3, 101301 (2000). 


\section{FIGURE CAPTIONS}

Fig. 1. The one-dimensional nonlinear wakefield excited by the linearly-polarized laser pulse with peak normalized amplitude $a_{0}=2, \sigma_{z}=2, \gamma=10.1$ - The normalized longitudinal electric field $E_{z}(\xi)$; 2 - the dimensionless potential of the wakefield $\Phi(\xi) ; 3$ - the amplitude of the laser pulse $a(\xi)$.

Fig. 2. Dependence of electron initial momentum $p_{0}$ on the trapping point near the first accelerating maximum. $1-a_{0}=2 ; 2-a_{0}=3$.

Fig. 3. The minimum momentum of the trapped electrons $p_{\min }$ (curve 1) and the wake wave amplitude $E_{z, \max }$ (curve 2) in dependence on peak amplitude of the laser pulse $a_{0}$.

Fig. 4. Trapping, compression and acceleration of initially mono-energetic electron bunch in the wakefield presented in Fig. $1, p_{0}=0.5,1 \leq \xi_{0} \leq 6$. Evolution of the coordinate (a) and the relativistic factor (b) of electrons.

Fig. 5. Behavior of electrons with initial position $\xi_{0}=0$ and with initial momentums $p_{0}=0.5,0.8,1$ and 1.2 in the wakefield shown in Fig. 1. Electrons with smaller initial momentums are trapped earlier. (a) Coordinate and (b) relativistic factor of electrons.

Fig. 6. The radial profiles of unperturbed electron density in the plasma channel (curve 1) and the laser pulse (curve 2), $r_{c h}=\sigma_{r}=5, b=0.01$.

Fig. 7. The two-dimensional nonlinear laser wakefield excited in the plasma channel with the radial density profile shown in Fig. $6, a_{0}=2, \sigma_{z}=2, \sigma_{r}=5$. (a) The longitudinal electric field for $r_{0}=0,3$ and 5 in the order of magnitude reduction. (b) The focusing field $f_{r}=\beta_{g} H_{\theta}-E_{r} .1-r=1 ; 2-r=3$; $3-r=5$.

Fig. 8. The radial behavior of the wakefield shown in Fig. 7, at $\xi=-10.9 .1$ - longitudinal electric field $E_{z}(\xi=-10.9, r) ; 2$ - the focusing force $f_{r}(\xi=-10.9, r)$.

Fig. 9. Trapping and acceleration of electrons with zero initial momentums in the wakefield given in Fig. 7, $p_{z 0}=0.8, \xi_{0}=0$. Longitudinal (a) and radial (b) positions and relativistic factor (c) of electrons.

Fig. 10. The characteristic dependence of the minimum trapping threshold on initial radial position of electron, $p_{r 0}=0, \xi_{0}=0$.

Fig. 11. Maximum (curve 1) and minimum (curve 2) initial radial momentums of trapped electrons depending on initial radial position, $p_{z 0}=0.8, \xi_{0}=0$.

Fig. 12. Trapping, compression and acceleration of an electron bunch in the wakefield presented in

Fig. 7. Initial parameters of the bunch are: $0 \leq \xi_{0} \leq 5, r_{0} \leq 4,0.6 \leq p_{z 0} \leq 0.8,-0.02 \leq p_{r 0} \leq 0.02$. Radial positions (a) and relativistic factor (b) of electrons. 
Figure 1.

A. G. Khachatryan

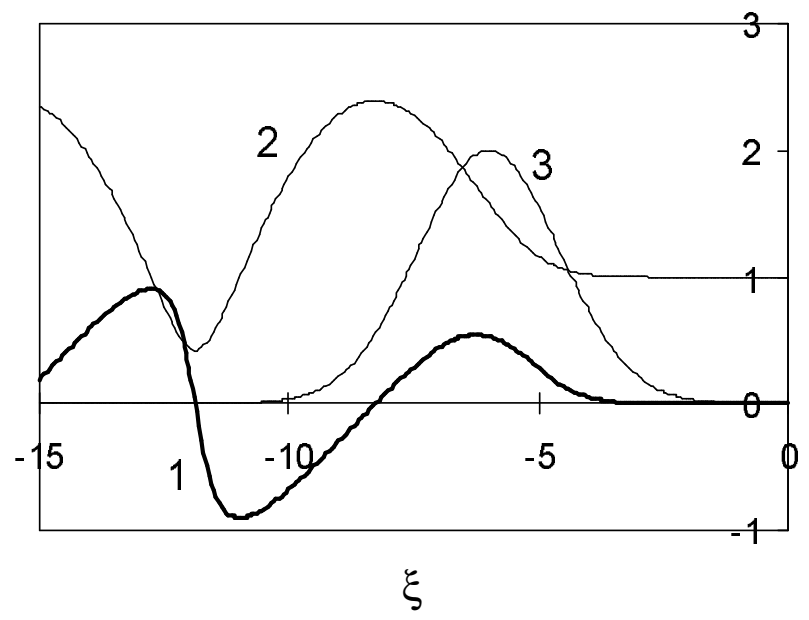


Figure 2.

A. G. Khachatryan

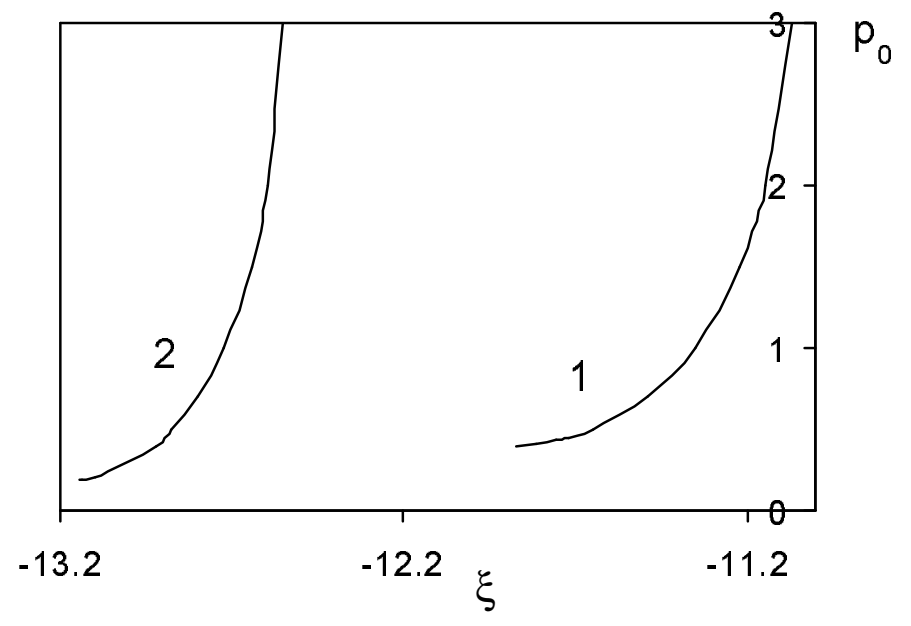


Figure 3.

A. G. Khachatryan

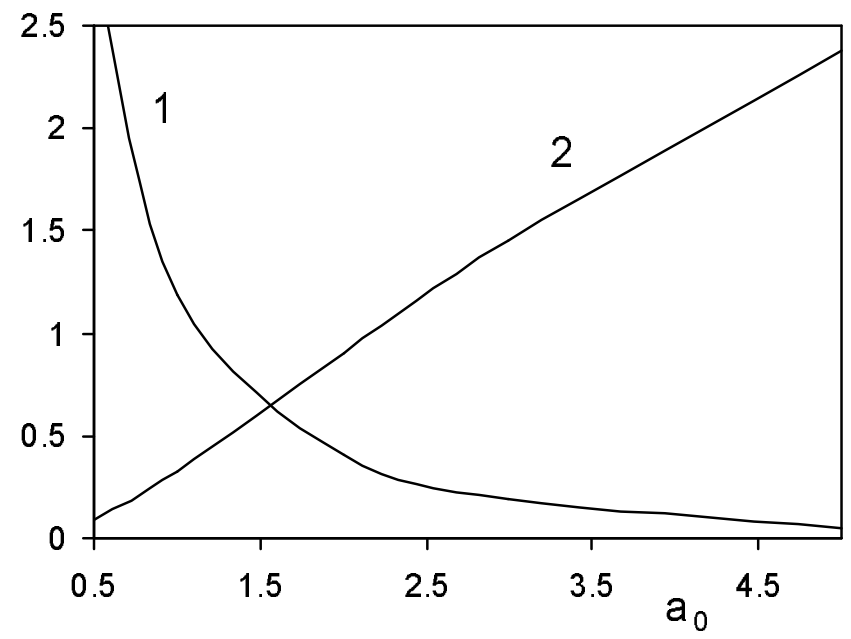


Figure 4.

A. G. Khachatryan
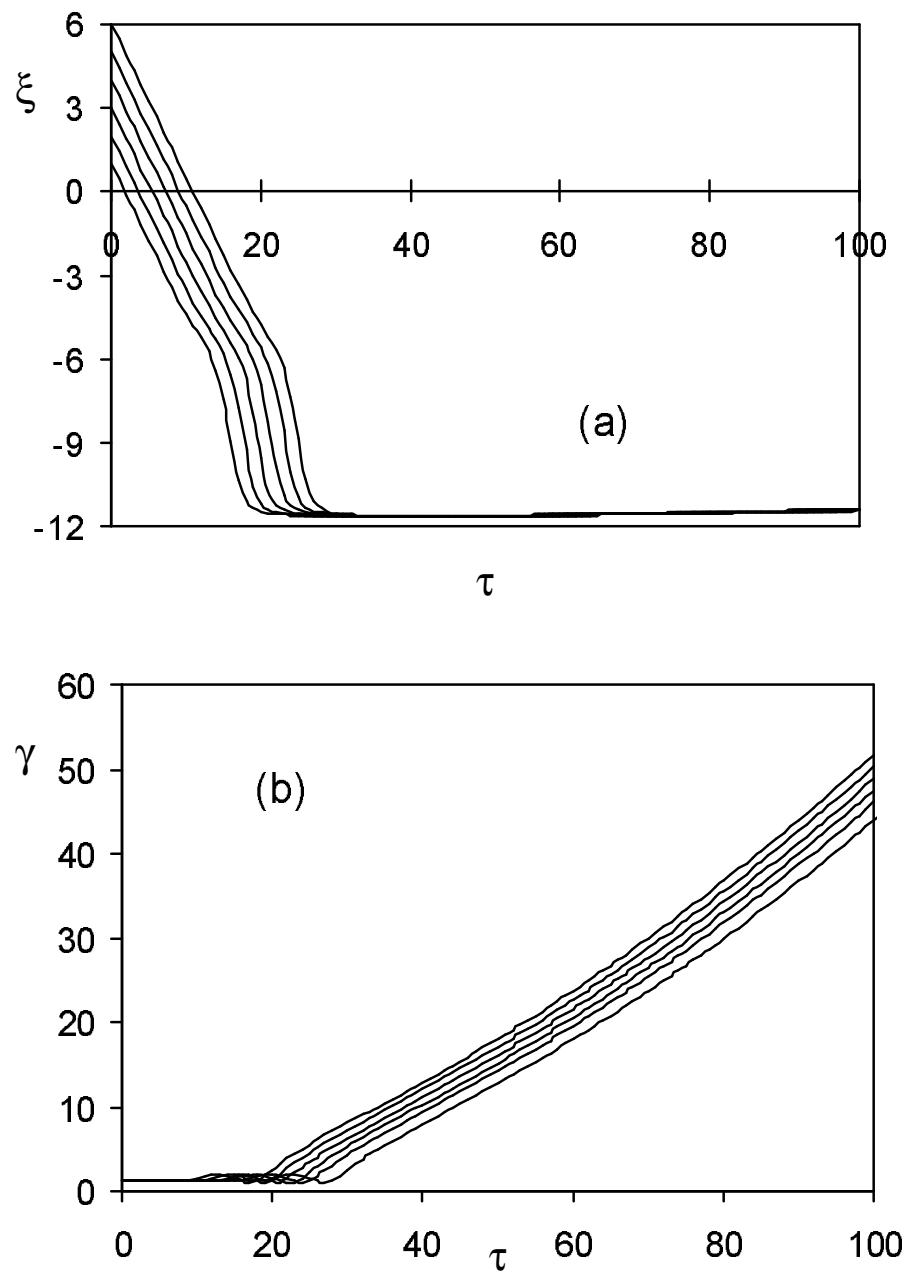
Figure 5.

A. G. Khachatryan
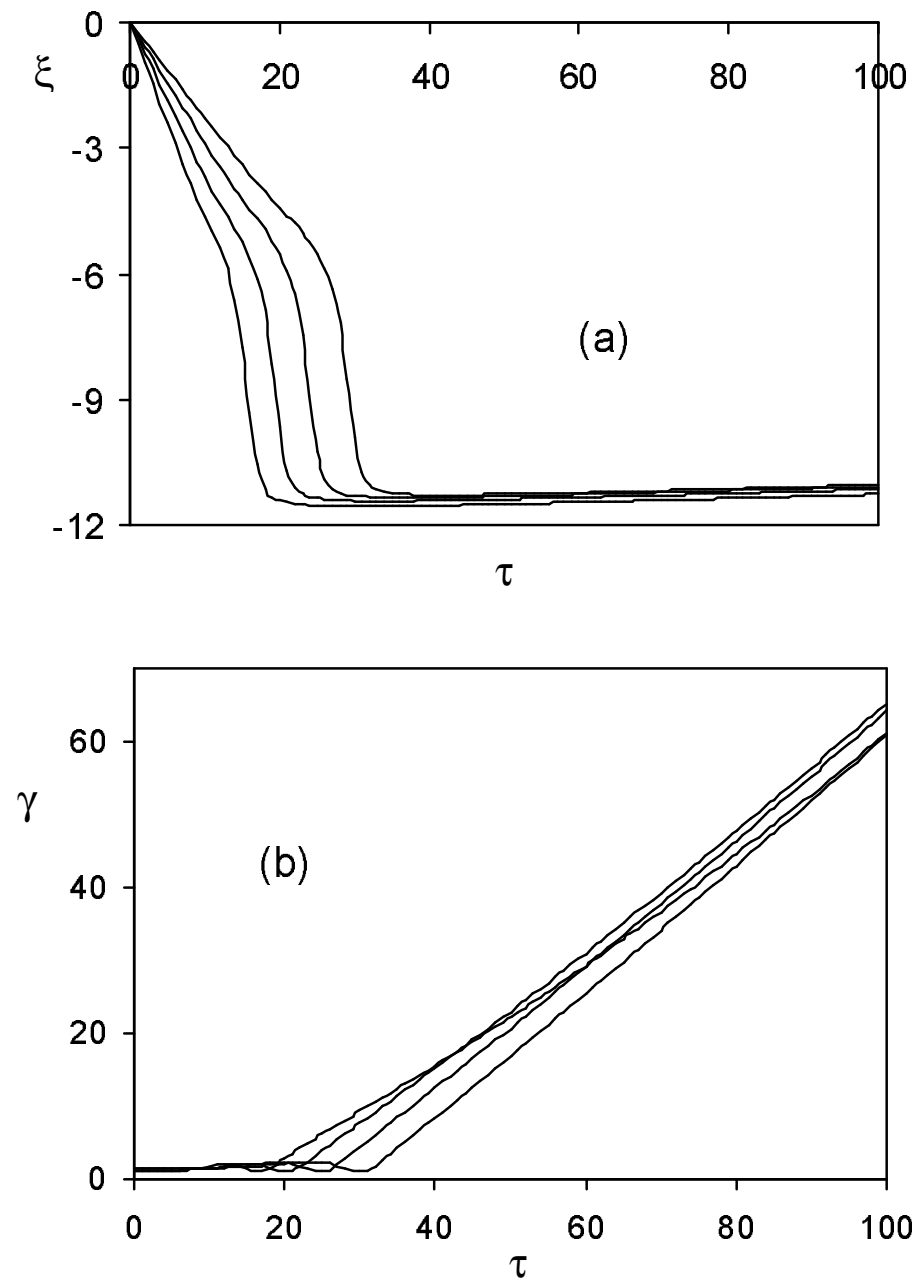
Figure 6.

A. G. Khachatryan

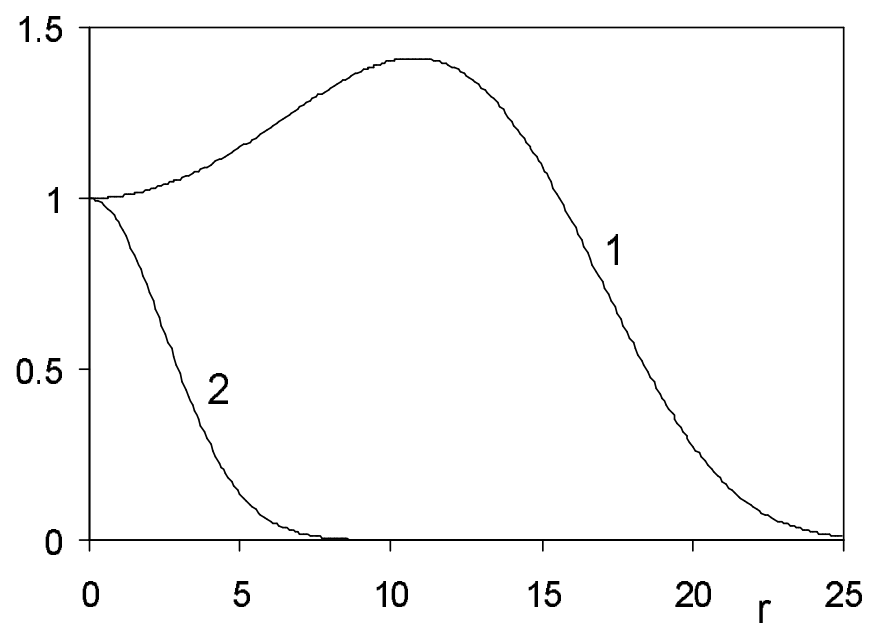


Figure 7.

A. G. Khachatryan
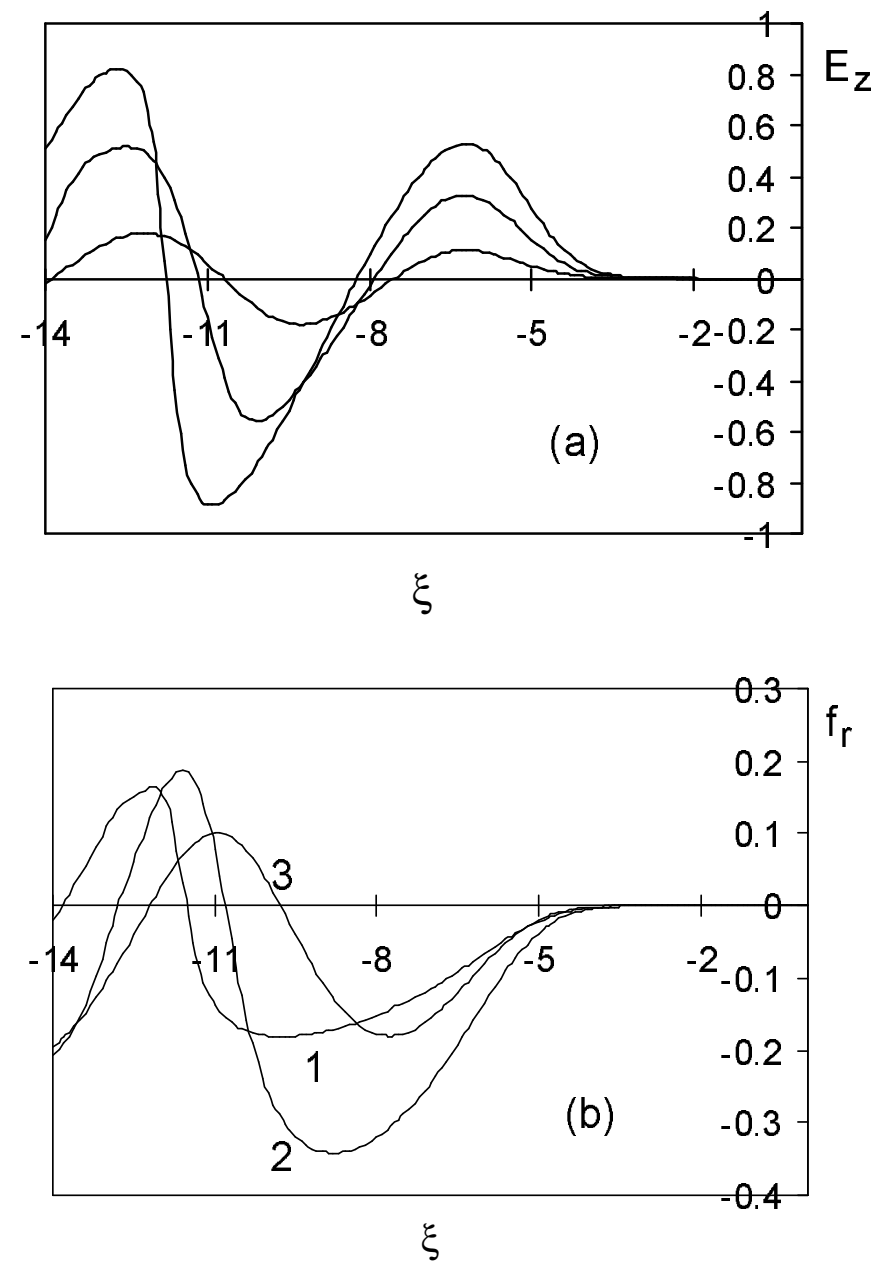
Figure 8.

A. G. Khachatryan

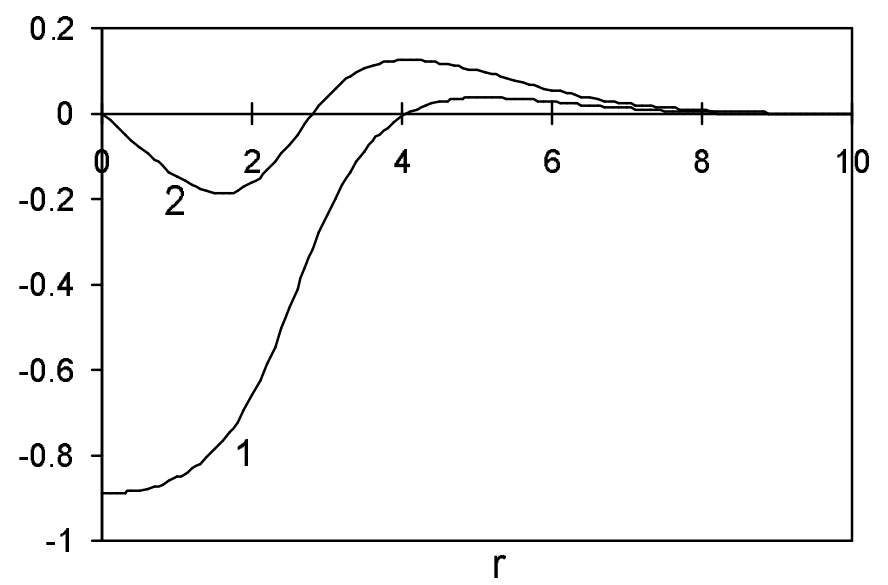


Figure 9.

A. G. Khachatryan
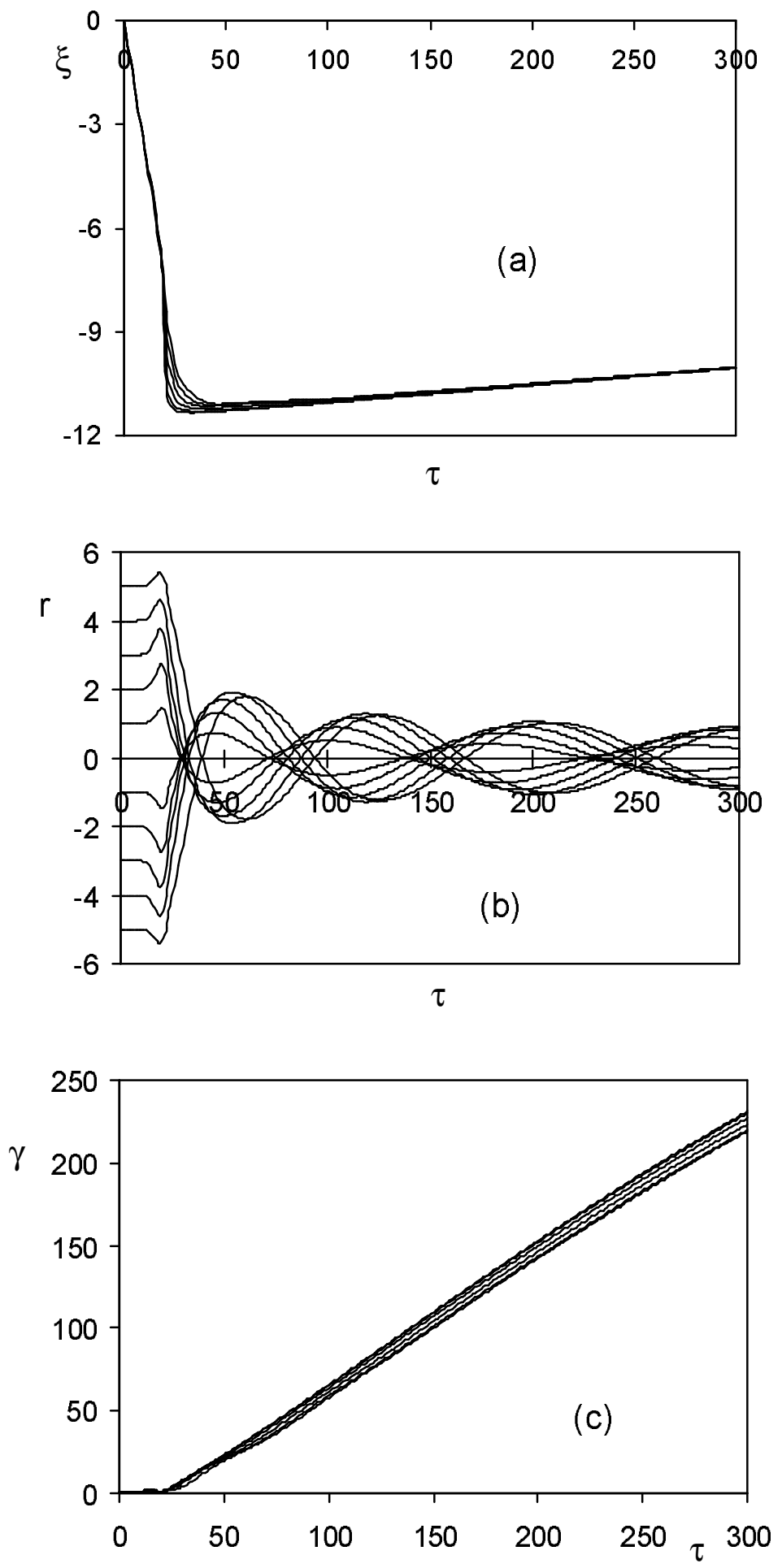
Figure 10.

A. G. Khachatryan

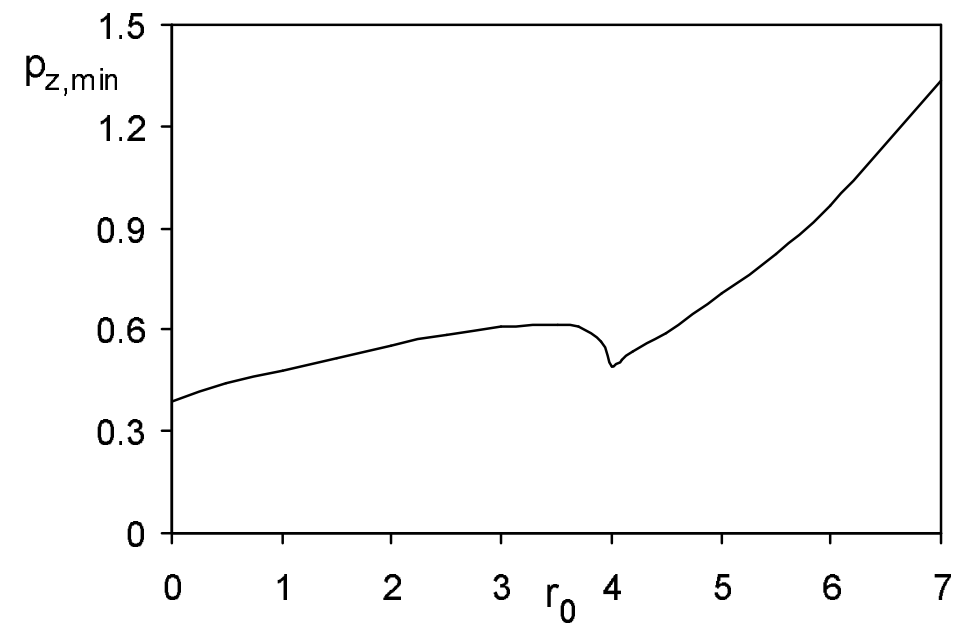


Figure 11.

A. G. Khachatryan

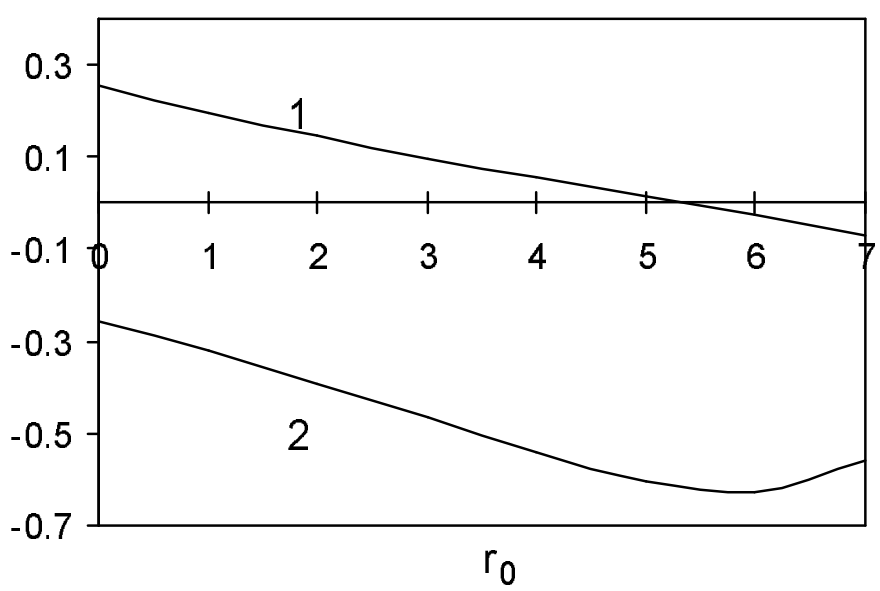


Figure 12.

A. G. Khachatryan
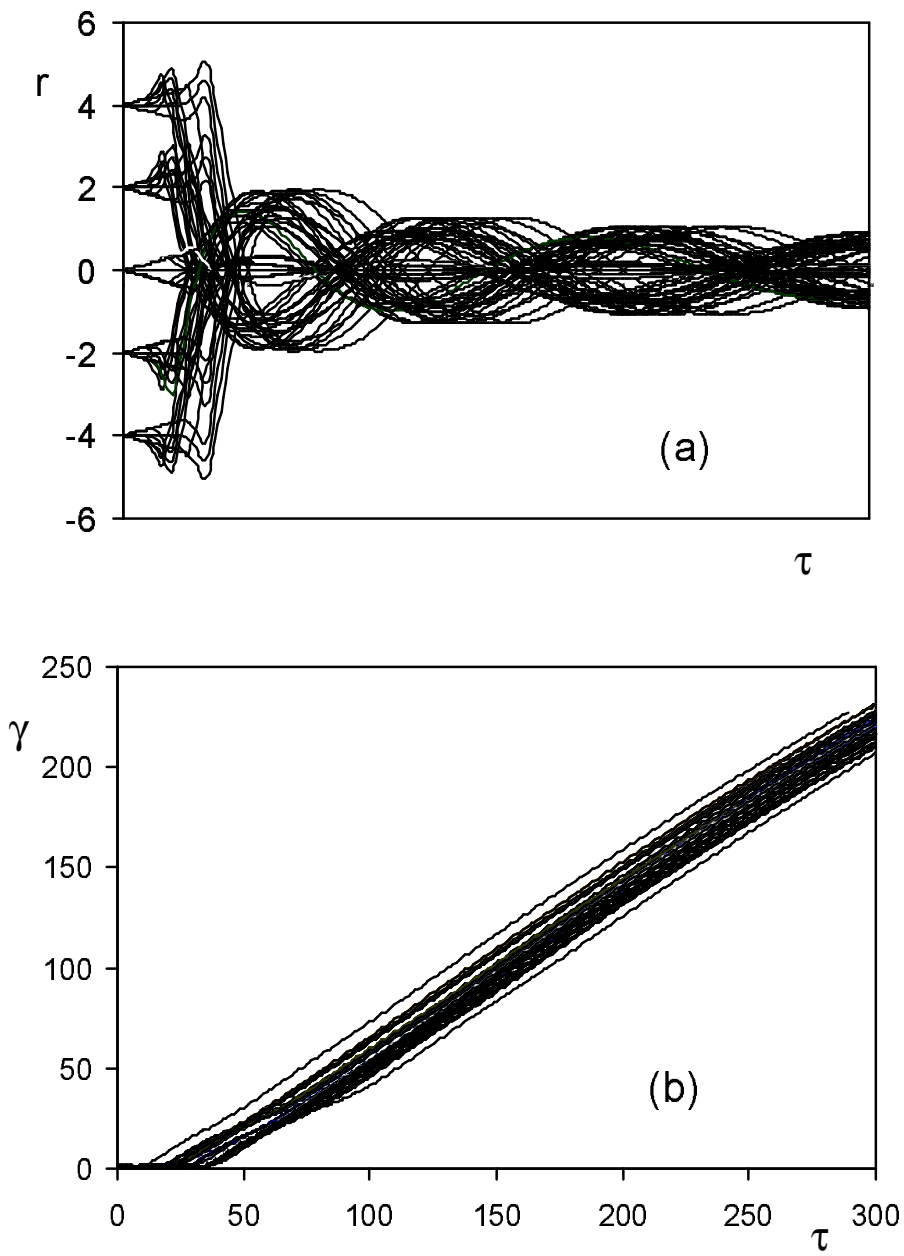